\title{
Landeskunde im DaF-Unterricht: kognitiv, kommunikativ, interkulturell oder diskursiv?
}

\author{
Alexandra NICOLAESCU \\ Lekt. Dr.; Universität Bukarest; \\ E-Mail: alexandra.nicolaescu@1ls.unibuc.ro
}

\begin{abstract}
Information about culture and civilization has always been an integrated part of the courses for German as a foreign language. In the past few decades were developed several theoretical approaches regarding that aspect, for example the cognitive, the communicative or the intercultural approach. In course books and learning materials the culture and civilization component is most of the times related with the linguistic component, but recently, influenced by the development of the field of cultural studies, the cultural discourse has also become a priority. In the following article my aim is to depict to what extent this paradigm shift on a theoretical level is being reflected in learning materials. While the first part of the article is dedicated to the theoretical aspect, in the second part I intend to analyze examples of exercises and worksheets with regard to the educational objective.
\end{abstract}

Keywords: teaching methods, German as a foreign language, culture and civilization, course books

\section{Vorbemerkungen}

Die Landeskunde ist ein wesentlicher Bestandteil des DaFUnterrichts, sodass im Laufe der Zeit unterschiedliche didaktische Ansätze, welche die Grundlagen der Vermittlung von 
Landeskunde darstellen, entwickelt wurden. Dazu gehören der kognitive, der kommunikative und der interkulturelle Ansatz. Da sich die Ziele und Inhalte des landeskundlichen Lernens verändern und Landeskunde eng mit dem Sprachgebrauch zusammenhängt, kann man in den aktuellen DaF-Materialien einen neuen Zugang zur Landeskunde ausmachen: den diskursiven Ansatz ${ }^{1}$. Wie wird dieser neue Ansatz definiert? Was setzt das diskursive landeskundliche Lernen voraus? Entsprechen die Lehrwerke, die wir im DaF-Unterricht einsetzen, dieser neuen Auffassung von Sprach- und Kulturvermittlung? Das sind die zentralen Fragen, auf die ich im Beitrag eingehen möchte, wobei ich im ersten Teil theoretische Aspekte erörtere und im zweiten Teil Auszüge aus den von mir im Unterricht eingesetzten Lehrbüchern analysiere, um die Vermittlung der Landeskunde im Unterrichtsalltag näher zu beleuchten.

\section{Theoretische Grundlagen}

\subsection{Der kognitive Ansatz}

In den 1950er-Jahren galt als übergeordnetes Lernziel des Fremdsprachenunterrichts das Vermitteln der Sprache selbst bzw. die Kenntnis des Sprachsystems. Landeskundliche Inhalte gab es in den Lehrwerken als Zusatzmaterialien in eigenständigen Lektionsabschnitten und der Bezug zur Praxis, die Anwendung der Fremdsprache, kam überhaupt nicht vor. Insofern ging es bei dem landeskundlichen Ansatz ausschließlich um Fakten und Informationen aus verschiedenen Disziplinen, folglich um einen kognitiven Ansatz, auch faktischer Landeskundeansatz genannt.

Der faktische Landeskundeansatz schöpft aus einer unendlichen Menge an Daten, Zahlen und Fakten jeder einzelnen Disziplin. Die Autoren von Lehrwerken bzw. die Lehrenden stehen also vor einer

${ }^{1}$ Dieser wird in der Fachliteratur auch als kulturwissenschaftlicher Ansatz bezeichnet. 
Vielzahl von landeskundlichen Informationen, aus denen einige wenige für den Unterricht ausgewählt werden müssen. ${ }^{2}$

Faktische Landeskunde ist dementsprechend nicht in den Sprachunterricht integriert, sondern die zu vermittelnden Informationen nehmen eine begleitende Rolle ein, wobei die Lernenden Kenntnisse in Form konkreter Informationen erwerben. Die Themen wurden vornehmlich aus den Bereichen Geschichte, Politikwissenschaft, Soziologie usw. abgeleitet und das Wissen in Form von Sachtexten, Statistiken, Tabellen oder Schaubildern präsentiert.LautderTheorieberuhtderfaktischeLandeskundeansatz auf einem traditionellen Kulturbegriff, der mit dem Bereich der Hochkultur gleichzusetzen ist und nicht auf dem erweiterten Kulturbegriff, der die gesamte Lebenswirklichkeit der in einem bestimmten Sprach- und Kulturraum lebenden Menschen und alle Produkte ihres Denkens und Handelns umfasst ${ }^{3}$ und sich erst zu Beginn der 1970er-Jahre durchgesetzt hat.

\subsection{Der kommunikative Ansatz}

In den 1970er-Jahren erfolgte eine Neuorientierung in der Fremdsprachendidaktik im Rahmen der Bildungsreform in Deutschland. Die Sprache wurde nicht mehr ausschließlich als System, sondern auch als Medium zur Kommunikation angesehen.

Das Interesse an Sprache verlagerte sich von der präzisen Beschreibung formaler Eigenschaften auf pragmatische Ziele, d.h., wie Menschen miteinander kommunizieren. Die Fähigkeit, sich in bestimmten Situationen mitteilen zu können, führte zu einer Auffassung von Sprache als menschlichem Handeln. ${ }^{4}$

Es ging ab diesem Zeitpunkt darum, dass Lernende in der Lage sind, ihre Sprechintentionen deutlich zu realisieren, um

2 Padrós, Alicia/Markus Biechele: Didaktik der Landeskunde. Fernstudieneinheit 31. München 2003, S. 23.

3 Vgl. ebd., S. 12.

${ }^{4}$ Ebd., S. 33. 
dadurch den Alltag im deutschsprachigen Raum zu bewältigen. Im Mittelpunkt der Vermittlung von landeskundlichem Wissen standen folglich bestimmte Handlungsabläufe, für die Redemittel angeboten werden müssen, wobei das Gelingen von sprachlichen Handlungen und das Verstehen alltagskultureller Phänomene die globalen Lernziele ausmachten. Politik, Geschichte und Soziologie als Bezugswissenschaften rückten in den Hintergrund. Die Relevanz der Alltagsthemen für eine angemessene Verwendung der Sprache stieg. Bei der Themenwahl wurde vorausgesetzt, dass die Lernenden mit bestimmten Situationen konfrontiert werden, die sie sprachlich auch bewältigen müssen. Der Einbezug von Texten, „die in der Alltagsrealität des Zielsprachenlandes tatsächlich vorkommen", war mit dem Bestreben verbunden, diese Sprachwirklichkeit „möglichst genau" abzubilden. ${ }^{5}$ In den Aussagen der Lernenden kommt es daher mehr auf den Inhalt als auf die grammatische Korrektheit an, sodass die Landeskunde dementsprechend als integrierter Bestandteil einer kommunikativen Didaktik betrachtet wurde.

\subsection{Der interkulturelle Ansatz}

Die Wahrnehmung stellt den Schlüsselbegriff in der interkulturellen Landeskunde dar, da beim Wahrnehmen und Interpretieren der Wirklichkeit stets auf eigene Erfahrungen, auf die eigene Umgebung und damit auch auf die eigene Kultur zurückgegriffen wird. Im Fremdsprachenunterricht lernt man neben der Sprache auch einen fremden Kulturkreis kennen und dementsprechend wird ein kontext- und situationsgerechtes Verstehen gefördert.

Wenn Wahrnehmung aber von unseren unterschiedlichen kulturellen und individuellen Erfahrungen geprägt wird, kann es passieren, dass wir Situationen, denen wir in einem anderen Land ausgesetzt

5 Ebd., S. 43. 
sind, anders interpretieren und verstehen, als sie möglicherweise gemeint sind. ${ }^{6}$

Es muss noch hinzugefügt werden, dass es dabei um sprachliche und nichtsprachliche Handlungen geht. Dementsprechend haben sich Anfang der 1980er-Jahre Vertreter der Fachdidaktik für Deutsch als Fremdsprache darum bemüht, beim Erstellen von Lernmaterialien auch die Perspektive der Lernenden einzubeziehen. Fragen zur Wahrnehmung rückten in den Mittelpunkt, sodass beim Deutschlernen eine doppelte Progression erfolgte: Einerseits ging es um die Sprachkenntnisse und andererseits um das kulturspezifische Wissen. Dadurch sollen sich die Lernenden Strategien aneignen, um fremde Bedeutungen zu erschließen, und für Unterschiede sensibilisiert werden. Der interkulturelle Ansatz verdeutlicht somit die Tatsache, dass der Kulturvergleich auch eine Auseinandersetzung mit der eigenen Kultur voraussetzt und dass die Fähigkeit der Lernenden zur Bewertung von zunächst unbekannten Kontexten geschult werden kann. Das Fremdverstehen und das Orientierungsvermögen gelten als Lernziele im Rahmen dieses Ansatzes.

\subsection{Der diskursive Ansatz}

Wie bisher dargestellt, haben sich die Ziele und Inhalte des landeskundlichen Lernens im Laufe der Zeit verändert und mussten immer wieder neu bestimmt und ausgehandelt werden. Heutzutage spricht man von einem kulturwissenschaftlichen landeskundlichen Begriff, der sich von dem interkulturellen Ansatz dadurch unterscheidet, dass man nicht mehr voraussetzt, Länder oder Kulturen könnten eindeutig voneinander abgegrenzt werden. Im Zeitalter der Globalisierung bewegen sich die Menschen nicht nur im geschlossenen Kulturraum ihrer Herkunftsregion, sie kommen über die elektronischen Medien und dank der Reisemöglichkeiten stets in Kontakt mit Vertretern anderer Kulturkreise. Davon ausgehend, spricht Claus Altmayer

${ }^{6}$ Ebd., S. 46. 
von einer diskursiven Aushandlung von Bedeutungszuschreibungen bzw. Wissensordnungen. Er geht davon aus, dass stabilisierte Wissensordnungen in Diskurssituationen automatisiert werden und dementsprechend in sprachlich-diskursiven Handlungen neu definiert werden: „Für die Landeskunde heißt das: Ihre Gegenstände sind nicht irgendwo in einer ,objektiv ${ }^{`}$ existierenden Welt zu suchen, ihre Gegenstände sind die vor allem sprachlichen Äußerungen, mit denen wir diese als auch soziale und gemeinsame Wirklichkeit überhaupt herstellen" ${ }^{\text {“7 }}$. Um seine These zu erläutern, nennt Altmayer Themen wie Essen und Trinken, Familie oder Gesundheit. Es geht nicht nur darum, was die Menschen in deutschsprachigen Ländern essen, sondern darum, wie sie darüber sprechen und schreiben, also welche Deutungsmuster damit verbunden sind:

Gegenstand der Landeskunde in dem Sinn einer ,diskursiven ${ }^{\text {" }}$ Landeskunde, wie wir sie vertreten, sind nicht Fakten oder kulturspezifische Verhaltensweisen, sondern Diskurse, die in Diskursen verwendeten Deutungsmuster und die auf dieser Basis diskursiv hergestellten und ausgehandelten Bedeutungen ${ }^{8}$.

Im Rahmen dieses landeskundlichen Ansatzes spielt der Aufbau einer Diskursfähigkeit eine wesentliche Rolle. Die Lernenden müssen in der Lage sein, an Bedeutungskonstruktionen mitzuwirken und diese zu erweitern und zu hinterfragen. Es geht dabei um die Bewusstmachungen der Pluralität von Diskurspositionen und um die Auseinandersetzung mit eigenen und neuen Deutungsmustern.

\subsection{Landeskunde und kulturelles Lernen}

Während man vor Jahrzehnten noch von faktischer Landeskunde sprach, dreht sich heutzutage alles um das kulturelle Lernen.

\footnotetext{
7 Altmayer, Claus (Hg.): Mitreden. Diskursive Landeskunde für Deutsch als Fremd- und Zweitsprache. Stuttgart 2016, S. 9.

8 Ebd.
} 
Inwiefern lohnt es sich aber in einer globalisierten Welt noch Landeskunde als separates Fach der Fremdsprachendidaktik zu betrachten? Dieser Frage versucht Claus Altmayer nachzugehen. Ausgehend von der Voraussetzung, dass alle Phänomene, mit denen sich die Landeskunde beschäftigt, auf nationalstaatlich und territorial gegliederten Ordnungskategorien beruhen, schlägt er einen Paradigmenwechsel von der Landeskunde zu den Kulturstudien vor.

Die herkömmliche Landeskunde [...] ist nicht nur begrifflich, sondern vor allem auch konzeptionell überholt. Auf die oben unter dem Stichwort der kulturellen Globalisierung skizzierten dramatischen Veränderungen und Verwerfungen, die die Welt des 21. Jahrhunderts und damit auch die Welt unserer Lerner prägen, vermag sie auf der Basis des ihr eigenen methodologischen Nationalismus keine Antwort mehr zu geben. ${ }^{9}$

In seinen Ausführungen knüpft er an das angloamerikanische Konzept der Cultural Studies an, die auch im Fach Deutsch als Fremd- und Zweitsprache in den letzten Jahren zu einer kulturwissenschaftlichen Transformation der Landeskunde geführt haben. Die direkte Folge dieser Veränderung bestand darin, dass sich die Perspektivierung der Gegenstände und Themen, mit denen sich die Landeskunde befasst, verschob. Es geht heutzutage nicht mehr um objektive Darstellungen von Sachbeständen aus dem deutschsprachigen Kulturkreis, sondern um Prozesse der Deutung dieser Sachbestände:

Diese Verschiebung des thematischen Fokus ist das Ergebnis der intensiven Auseinandersetzung mit dem, was in der internationalen kulturwissenschaftlichen Debatte der letzten Jahre und Jahrzehnte als cultural turn bezeichnet wird. ${ }^{10}$

9 Altmayer, Claus: Landeskunde im Globalisierungskontext: Wozu noch Kultur im DaF-Unterricht. In: Haase, Peter/Michaela Höller (Hgg): Kulturelles Lernen im DaF-/DaZ-Unterricht. Paradigmenwechsel in der Landeskunde. Göttingen 2017, S. 3-22; hier S. 10.

${ }^{10}$ Ebd., S. 11. 
Altmayer geht mit der Kritik an dem Konzept des landeskundlichen Lernens sogar noch einen Schritt weiter und meint, dass sich in dem aktuellen theoretischen Zusammenhang der Begriff der Landeskunde als obsolet erweist. Beim Fremdsprachenlernen sollte ausschließlich über das kulturelle Lernen oder kulturbezogene Lernen diskutiert werden. Die Fähigkeit der Lernenden zur Diskurspartizipation sollte dabei als Lernziel definiert und damit die Möglichkeit zur globalen Interaktion gefördert werden. Es wäre noch wichtig zu erwähnen, dass die Lernenden nicht als Vertreter einen Nationalkultur, die automatisch aufgrund ihrer Herkunft über bestimmte Deutungsressourcen verfügen, angesehen werden dürfen. Damit das kulturelle Lernziel auch erreicht werden kann, werden Lernende im Rahmen der Kulturstudien als Individuen in ihren potenziell heterogenen sozialen und kulturellen Kontexten wahrgenommen, die innerhalb von lokalen, auch überregionalen, globalen, familien- und milieuspezifischen Deutungsebenen agieren.

\subsection{Das DACH-Prinzip}

Man kann nicht von theoretischen Ansätzen in der Didaktik der Landeskunde für Deutsch als Fremd- und Zweitsprache sprechen, ohne das DACH-Prinzip zu erwähnen. Im Folgenden möchte ich knapp darstellen, was dieses Prinzip voraussetzt. Das DACH-Prinzip hat eine sprachenpolitische Grundlage, ist auf institutioneller Ebene verankert und beruht auf einer Kooperationsbasis, die in der landeskundlichen Unterrichtspraxis, in Lehrwerken und in der Prüfungsgestaltung umgesetzt wird. Es geht dabei um die Einbeziehung des gesamten deutschsprachigen Raumes im DaF-Unterricht, was seit mehreren Jahren die landeskundlichen Lerninhalte prägt. Der DACH-Fokus „erlaubt es, verschiedene sprachenpolitische, landeskundliche, kulturwissenschaftliche und unterrichtspraktische Sichtweisen auf Deutsch als Fremdsprache immer wieder ins Gespräch zu 
bringen"11. Je nach Lernort und Lernperspektive sollte man unter Berücksichtigung des DACH-Prinzips das meist unreflektierte Vorwissen der Lernenden über den deutschsprachigen Raum zu einer systematischen Kenntnisgrundlage weiterentwickeln. Die Lehrwerke spielen in diesem Zusammenhang eine zentrale Rolle, da sie den Lehrenden die Mittel zu einer integrativen und reflexiven Landeskundevermittlung zur Verfügung stellen, wobei die binnenkontrastive wie auch die grenzüberschreitende Perspektive in den Unterricht einbezogen werden müsste.

Angesichts der Tatsache, dass im Rahmen des aktuellen kulturwissenschaftlichen Diskurses eine nationalstaatliche Perspektive als unangemessen gilt, wurde auch das DACH-Konzept hinterfragt und kritisiert. In den letzten theoretischen Veröffentlichungen steht fest: „Weder das D-A-CH-Konzept noch das DACH-Prinzip plädieren ausschließlich für etwas, das sich am besten unter dem Begriffsungetüm einer ,Kulturcontainerraumlandeskunde" subsumieren lässt." ${ }^{\text {"12 }}$ Dementsprechend lassen sich kein national definierter Raum oder nationalstaatliche Ordnungskategorien ableiten. Als Zielsetzung gilt ein kulturkontrastives Vorgehen, das zur Entwicklung einer DACHDiskursfähigkeit ${ }^{13}$ beitragen sollte.

${ }^{11}$ Krumm, Hans-Jürgen: Das Dach-Prinzip im Fach Deutsch als Fremdund Zweitsprache: Rück- und Ausblicke. In: Schafer, Naomi/Annegret Middeke/Sara Hägi-Mead et al.: Weitergedacht. Das DACH-Prinzip in der Praxis. Göttingen 2020, S. 3-12; hier S. 5.

${ }^{12}$ Tonsern, Clemens: Die Grenzen des Diskurses: Zu den Möglichkeiten einer kulturwissenschaftlichen Grundlegung des DACH-Prinzips. In: Schafer, Naomi/Annegret Middeke/Sara Hägi-Mead et al.: Weitergedacht. Das DACH-Prinzip in der Praxis. Göttingen 2020, S. 23-35; hier S. 27.

${ }^{13}$ Vgl. ebd., S. 28. 


\section{Landeskunde in DaF-Lehrwerken und Lernmaterialien}

Im Folgenden möchte ich anhand konkreter Beispielen darstellen, inwiefern ältere und neuere Ansätze zum landeskundlichen Lernen in Lehrwerken und Lernmaterialien eingebracht sind. Im Lehrwerk DaF kompakt neu für die Niveaustufen A1 - B1 lässt sich ein kognitiver Ansatz ausmachen, obwohl dieses Lehrwerk 2017 in überarbeiteter Fassung neu erschienen ist. Im letzten Kapitel der Niveaustufe A1 gibt es einen Text über den Maler Paul Klee ${ }^{14}$ und die Fragen dazu fokussieren eindeutig auf Fakten: Die Lernenden müssen nämlich die biografischen Angaben aus dem Text herausfinden und aufschreiben. Tatsächlich erscheint diese Übung in einem Kapitel, das auch kommunikative Übungen mit dem Schwerpunkt „Schweiz“ beinhaltet. Hier allerdings handelt es sich jedoch um einen Sachtext und einen Ansatz, welcher der kognitiven Landeskunde zugeschrieben werden kann.

Auch in DaF kompakt neu gibt es auf Niveau A2 ein Kapitel, das der Stadt Köln gewidmet ist. Hier erscheinen relativ viele Höraufgaben, welche die Lernenden mit dem Kölscher Dialekt vertraut machen sollen, sowie Lesetexte, die über die Erfahrungen eines österreichischen Studenten in Köln berichten. Es ist jedoch auch eine Übung vorhanden, die eindeutig faktische Landeskunde als Schwerpunkt aufweist. Nach der Lektüre eines kurzen Textes zum Kölner Dialekt ${ }^{15}$ müssen die Lernenden einen Lückentext mit konkreten Informationen ergänzen. Dabei handelt es sich lediglich um Tatsachen und diese geschlossene Übung weist keine kommunikativen, interkulturellen oder diskursiven Lernziele auf. Es ist demnach festzustellen, dass der

\footnotetext{
${ }^{14}$ Braun, Birgit/Margit Doubek/Nadja Fügert et al.: DaF kompakt neu A1 - B1. Stuttgart 2016, S. 77.

${ }^{15}$ Ebd., S. 100.
} 
kognitive Ansatz in den Unterrichtsmaterialien sporadisch immer noch präsent ist.

In DaF kompakt neu gibt es allerdings auch zwei äußerst repräsentative Beispiele für den kommunikativen Ansatz. In Kapitel 7 (Niveau A1) kommt eine Anzeige zu einem Waschsalon ${ }^{16}$ vor. Rumänische Lernende werden hier mit einem alltagskulturellen Phänomen konfrontiert, das sie aus dem eigenen Kulturraum nicht kennen. Die Lernenden werden aufgefordert, sich im Waschsalon zu verabreden, folglich konkrete Redemittel zu gebrauchen, um das konkrete Umsetzen sprachlichen Handelns zu fördern.

Dasselbe Lernziel gilt auch für eine weitere Unterrichtssequenz aus DaF kompakt neu, diesmal auf Niveau A2. Im Kapitel 10 geht es nämlich um das Thema „Wohnungssuche“. Nachdem die Lernenden Wohnungsanzeigen aus Zürich ${ }^{17}$ lesen und die wichtigsten Informationen herausfiltern, müssen sie Wohnungsanzeigen formulieren und kurze Gespräche führen. In den Gesprächen übernehmen die Lernenden entweder die Rolle des Vermieters oder die des zukünftigen Mieters. Im Rahmen dieser Unterrichtssequenz wird folglich vorausgesetzt, dass sich die Lernenden mit dieser Situation später konfrontieren werden und diese sprachlich auch bewältigen müssen, was durchaus den kommunikativen didaktischen Lernzielen entspricht.

Im Lehrwerk Menschen (2012) lassen sich eindeutige Beispiele für den interkulturellen Ansatz finden. Die Lektion 15 (Niveau A1) hat als Schwerpunkt das Thema „Leben in der Stadt". Der Lesetext besteht aus einem Blogeintrag einer Bewohnerin des Stadtviertels Giesing aus München ${ }^{18}$. Die Person beschreibt, warum gerade Giesing ihr Lieblingsviertel ist. Nach

\footnotetext{
${ }^{16}$ Ebd., S. 65.

${ }^{17}$ Ebd., S. 89.

${ }^{18}$ Evans, Sandra/Angela Pude/Franz Specht: Menschen A1. Deutsch als Fremdsprache. Kursbuch. Ismaning 2012, S. 84.
} 
der Textlektüre müssen die Lernenden ihr Lieblingsviertel beschreiben bzw. argumentieren, warum sie gerne in diesem Stadtviertel leben. Somit müssen sich die Lernenden auf die eigene Umgebung und auf eigene Erfahrungen beziehen, was einem interkulturell-kontrastiven Ansatz entspricht.

In diesem Lehrwerk gibt es in weiteren Kapiteln Sequenzen, die als Projekt Landeskunde betitelt sind, und die landeskundliche Informationen präsentieren, mit denen sich die Lernenden aktiv auseinandersetzen müssen. Die Unterrichtssequenz, auf die ich mich beziehe, geht von einem Text über das Wetter in der Schweiz ${ }^{19}$ aus, wobei die Lernenden aufgefordert werden, das Wetter im eigenen Heimatland als Projektarbeit vorzustellen. FürEuropäermag diese Aufgabe wohl keineSchwierigkeiten bedingen. Wenn man jedoch bedenkt, dass das Lehrwerk weltweit eingesetzt wird, so ist ersichtlich, dass mit dem Erschließen von Unterschieden zwischen Kulturräumen ein interkultureller Ansatz verfolgt wird.

Um den diskursiven Ansatz zu illustrieren, eignet sich Claus Altmayers Materialsammlung Mitreden. Diskursive Landeskunde für Deutsch als Fremd- und Zweitsprache (2016), die neben den theoretischen Ausführungen auch Lernmaterial für die Niveaustufen A2, B1 und B2 beinhaltet. Das A2-Modul trägt den Titel Menschen und im dritten Kapitel geht es um die Rollenbilder von Männern und Frauen. In der ersten Kopiervorlage werden die Lernenden durch vorgegebene Redemittel aufgefordert, über körperliche Merkmale, Charaktereigenschaften und typische geschlechtsorientierte Aufgaben zu diskutieren. ${ }^{20}$ Dadurch werden Wortschatz und Diskursfähigkeit aus kulturspezifischer Sicht trainiert. Um die Aufgabe zu lösen,

${ }^{19}$ Habersack, Charlotte/Angela Pude/Franz Specht: Menschen A2. Deutsch als Fremdsprache. Kursbuch. Ismaning 2013, S. 105.

${ }^{20}$ Altmayer, Claus: Die DACH-Landeskunde im Spiegel aktueller kulturwissenschaftlicher Ansätze. In: Demmig, Silvia/Sara Hägi/ Hannes Schweiger (Hgg.): DACH-Landeskunde. Theorie Geschichte - Praxis. München 2013, S. 15-31; hier S. 22. 
müssen die Lernenden die eigenen Deutungsmuster reflektieren und in der Interaktion ihre Meinungen aushandeln. Der Diskussion geschlechtsspezifischer Rollenbilder folgen zwei Werbespots zum Thema „Frauen zu Hause“. Der erste, ältere Werbespot zeigt das traditionelle Bild einer Hausfrau, die sich tagsüber ausschließlich um den Haushalt kümmert. Es wird behauptet, dass sich eine Frau im Leben nur darüber Gedanken machen sollte, was sie kochen oder anziehen soll ${ }^{21}$. Der zweite Werbespot stellt eine „moderne“ Hausfrau dar, diesmal als erfolgreiche Familienmanagerin dargestellt, zu deren Aufgaben die Nachwuchsförderung, die Kommunikation und die Mitarbeitermotivation ${ }^{22}$ gehören. Hier spielt der Wortschatz eine wesentliche Rolle, da über das Leben und die Aufgaben einer Hausfrau diskutiert werden soll und nicht über die Rolle der Hausfrau selbst. Demzufolge dient hier Sprache vor allem dazu, einer bestimmten Weltauffassung Aussagen zuzuweisen. Beim Vergleich der zwei Werbespots erwerben die Lernenden die Fähigkeit, themenbezogene diskursive Praktiken zu verstehen und durch persönliche Meinungsäußerungen zu trainieren.

\section{Schlussbetrachtungen}

In den letzten Jahrzehnten haben sich die theoretischen Grundlagen zur Vermittlung von landeskundlichen Informationen im DaF-Unterricht wesentlich geändert. Man spricht von einem Paradigmenwechsel, was die Schwerpunkte des landeskundlichen Lernens betrifft. Fakten sind längst nicht mehr von grundlegender Bedeutung, die kommunikative Wende macht das sprachliche Handeln zum Lernziel. Später trat das kulturwissenschaftliche Lernen in den Vordergrund, wobei das Verstehen fremder Diskurse im Fokus steht. In den gängigen Lehrbüchern lassen sich allerdings diverse theoretische Ansätze finden. Die

${ }^{21} \mathrm{https}: / /$ www.youtube.com/watch?v=072LrlGvSq8; 31.05.2021.

${ }^{22} \mathrm{https}: / /$ www.youtube.com/watch?v=h33F7YDqXM4; 31.05.2021. 
landeskundlichen Lernziele variieren von Lektion zu Lektion und man kann eigentlich keine klaren Grenzen zwischen den theoretischen Konzepten ziehen. Daher kommt der Lehrkraft eine wichtige Rolle zu, da diese über Lernziele entscheidet und unter Berücksichtigung der Bedürfnisse der Zielgruppe auch das Herangehen an Unterrichtssequenzen plant.

\section{Literaturverzeichnis}

\section{Lehrwerke und Lernmaterialien}

Altmayer, Claus (Hg.): Mitreden. Diskursive Landeskunde für

Deutsch als Fremd-und Zweitsprache. Stuttgart 2016.

Braun, Birgit/Margit Doubek/Nadja Fügert et al.: DaF kompakt neu A1-B1. Stuttgart 2016.

Evans, Sandra/Angela Pude/Franz Specht: Menschen A1.

Deutsch als Fremdsprache. Kursbuch. Ismaning 2012.

Habersack, Charlotte/Angela Pude/Franz Specht: Menschen A2. Deutsch als Fremdsprache. Kursbuch. Ismaning 2013.

\section{Sekundärliteratur}

Altmayer, Claus: Landeskunde im Globalisierungskontext: Wozu noch Kultur im DaF-Unterricht. In: Haase, Peter/ Michaela Höller (Hgg): Kulturelles Lernen im DaF-/DaZUnterricht. Paradigmenwechsel in der Landeskunde. Göttingen 2017, S. 3-22.

Altmayer, Claus: Die DACH-Landeskunde im Spiegel aktueller kulturwissenschaftlicher Ansätze. In: Demmig, Silvia/ Sara Hägi/Hannes Schweiger(Hgg.): DACH-Landeskunde. Theorie - Geschichte - Praxis. München 2013, S. 15-31.

Krumm, Hans-Jürgen: Das Dach-Prinzip im Fach Deutsch als Fremd- und Zweitsprache: Rück- und Ausblicke. In: Schafer, Naomi/Annegret Middeke/Sara Hägi-Mead et al.: Weitergedacht. Das DACH-Prinzip in der Praxis. Göttingen 2020, S. 3-12. 
Padrós, Alicia/Markus Biechele: Didaktik der Landeskunde. Fernstudieneinheit 31. München 2003.

Tonsern, Clemens: Die Grenzen des Diskurses: Zu den Möglichkeiten einer kulturwissenschaftlichen Grundlegung des DACH-Prinzips. In: Schafer, Naomi/Annegret Middeke/Sara Hägi-Mead et al.: Weitergedacht. Das DACH-Prinzip in der Praxis. Göttingen 2020, S. 23-35.

\section{Internetquellen}

https://www.youtube.com/watch?v=072LrlGvSq8;31.05.2021. https://www.youtube.com/watch?v=h33F7YDqXM4; 31.05.2021. 